\title{
Diacronie
}

Studi di Storia Contemporanea

$N^{\circ} 24,4 \mid 2015$

Le dittature militari: fisionomia ed eredità politica

\section{Autoritarismes civils et militaires en Corée du Sud:} 1948-1979

Laurent Quisefit

\section{(2) OpenEdition}

Journals

Édition électronique

URL : http://journals.openedition.org/diacronie/3854

DOI : 10.4000/diacronie.3854

ISSN : 2038-0925

Éditeur

Association culturelle Diacronie

Référence électronique

Laurent Quisefit, « Autoritarismes civils et militaires en Corée du Sud: 1948-1979 », Diacronie [En ligne], $N^{\circ} 24,4$ | 2015, document 18, mis en ligne le 29 décembre 2015, consulté le 20 avril 2019. URL : http:// journals.openedition.org/diacronie/3854; DOI : 10.4000/diacronie.3854 


\section{Diacronie}

N. 24 | 4|2015 Le dittature militari: fisionomia ed eredità politica

\section{8/}

\section{Autoritarismes civils et militaires en Corée du Sud: 1948-1979}

\section{Laurent QUISEFIT *}

En 1961, le coup d'Etat du général Park Chung-hee (1917-1979) amorce un processus de modernisation de la Corée du Sud. Après le clientélisme de Syngman Rhee (président de 1948 à 1960), et un an de flottement politique du cabinet Chang Myŏn, le régime militiaire est animé par des idéaux de réforme et de lutte contre la corruption. Pourtant, anti-communisme et routine aidant, cette réforme forcée aboutit à la mise en place d'un régime conservateur reproduisant les vices du système de Syngman Rhee. Malgré la perte des libertés individuelles, le volontarisme économique du général Park crée les bases du "miracle économique » coréen. Aussi, les sentiments à l'égard de cette dictature sont-ils ambivalents : rejet viscéral par les intellectuels citadins, quasi adulation dans certains milieux modestes ou ruraux. Freiné par les chocs pétroliers, le développement économique et la modernisation des campagnes, qui ne fondent la puissance économique de la Corée d'aujourd'hui, dessisnent le bilan ambivalent d'une dictature parfois féroce, dont le contexte de guerre froide explique certains travers. Dans le long terme, la dictature militaire ne prend fin qu'en 1987. Encore faut-il attendre 1993 pour voir élu le premier président civil. Nous entendons ici comparer différents aspects des régimes de Park et de Rhee, pour montrer que le pouvoir civil a pu s'avérer tout aussi tyrannique que le militaire, sans toujours parvenir à des résultats aussi visibles. Nous entendons ici explorer deux aspects de ces régimes, à savoir la dimension autoritaire et répressive d'une part, la question économique d'autre part 
La tyrannie la plus violente sert toujours la nécessité de voiler ses noirs desseins sous des formes légales.

Louis Philippe, comte de Ségur (1753-1830)

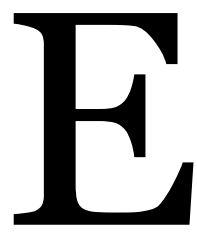

n 1961, le coup d'Etat du général Park Chung-hee' ${ }^{1}$ (1917-1979) ouvre un processus de modernisation de la Corée du Sud. Après le clientélisme de Syngman Rhee ${ }^{2}$ (président de 1948 à 1960), et un an de flottement politique du cabinet Chang Myŏn, le régime militiaire est animé par des idéaux de réforme et de lutte contre la corruption. Pourtant, anti-communisme et routine aidant, cette réforme forcée aboutit à la mise en place d'un régime conservateur reproduisant les vices du système de Yi Sŭngman. Malgré la perte des libertés individuelles, le volontarisme économique du général Park crée les bases du « miracle économique » coréen. Aussi, les sentiments à l'égard de cette dictature sont-ils ambivalents : rejet viscéral par les intellectuels citadins, quasi adulation dans certains milieux modestes ou ruraux. Freiné par les chocs pétroliers, le développement économique et la modernisation des campagnes, qui fondent la puissance économique de la Corée d'aujourd'hui, dessisnent le bilan ambivalent d'une dictature parfois féroce, dont le contexte de guerre froide explique certains travers. Dans le long terme, la dictature militaire ne prend fin qu'en 1987. Encore faut-il attendre 1993 pour voir élu le premier président civil.

Nous entendons ici comparer différents aspects des régimes de Park et de Yi, pour montrer que le pouvoir civil a pu s'avérer tout aussi tyrannique que le militaire, sans toujours parvenir à des résultats aussi visibles. Nous envisagerons ci-après deux aspects de ces régimes, à savoir la dimension autoritaire et répressive d'une part, la question économique d'autre part.

\section{Répressions}

Si les régimes de Yi Sŭngman et de Park Chung-hee se sont montrés répressifs, le contexte et les modalités de l'action coercitives sont assez différents. Aux urgences nées avec les insurrections et la guerre de Corée répond une pratique discrétionnaire du temps de paix qui semble plus mesurée sous les mandatures de Park Chung-hee.

\footnotetext{
${ }^{1}$ Nous nous conformons ici à l'usage coréen, où le patronyme précède le prénom.

2 Ci-après Yi Sŭngman.
} 


\subsection{La présidence de Yi Sŭngman}

Yi Sŭngman (1875-1965) est porté au pouvoir par les élections qui, à l'été 1948, entérinent la fondation de la République de Corées3. La situation de la Corée est difficile. D’une part, la péninsule est divisée, à partir du 9 septembre, en deux Etats concurrents, l'un communiste au Nord, l'autre capitaliste, au Sud. Ces deux entités coréennes excipent d'une même légitimité sur la totalité de la péninsule de Corée ${ }^{4}$.

La République de Corée est alors confrontée non seulement à une grave crise économique, mais encore aux retards pris par la réforme agraire. L’inflation, aggravée par l'incompétence du gouvernement de Yi Sŭngman en matière financière ${ }^{5}$, grève lourdement les dépenses des particuliers. L'économie ne s'est encore relevée ni de la division ni de la rupture des relations avec le Japon: la première a brisé la complémentarité entre un Nord industrialisé et un sud agricole, la secondé a interrompu tout commerce avec le Japon. La Corée manque de techniciens qualifiés, de spécialistes confirmés et même le gouvernement fait preuve d'un grand amateurisme.

C'est dans ce contexte que se développe une forte contestation sociale. Réclamant des hausses de salaire et de meilleures conditions de travail, ouvriers et employés défilent et font grève. La répression est féroce. Entre le 4 septembre 1948 et le 30 avril 1949, 89.710 personnes sont arrêtées, dont cinq journalistes et six membres de l'Assemblée nationale. De plus, dix-sept publications sont interdites sous prétexte de critiques anti-gouvernementales ${ }^{6}$. Un rapport de la Commission des Nations Unies pour la Corée, daté du 15 décembre 1949 rendait ainsi compte des opérations policières en Corée du Sud :

En 1949, il y a eu cent dix-huit mille six cent vingt et une arrestation entraînant soixante-huit mille deux cent sept inculpations. Pendant les quatre premiers mois de 1950, il y a eu trente-deux mille dix-huit arrestations. Certaines de ces arrestations ont entraîné des violations des libertés constitutionnelles et même des brutalités et des tortures7.

Un diplomate français en poste en Corée en 1948 qualifiait d'ailleurs la Corée du Sud de « régime hitlérien ». L'usage de la torture, le recours à des « organisations de

3 Fondée le 15 août 1948.

4 Au Nord, Pyongyang n'est alors considérée que comme une capitale temporaire.

5 KIM, Dong-uk, 1940-1950 nyŏndae Han'guk üi imp'ūlleisyŏn kwa anjŏnghwa chŏngch'aek, Thèse de doctorat, Université Yonsei, Seoul, 1995, p. 52.

${ }^{6}$ Voir HU Hung-lick, Le problème coréen, Paris, Pedone, 1953. p. 114.

7 Rapport de la Commission des Nations-Unies pour la Corée, 1949, vol. I, pp. 34-35. 
jeunesse ${ }^{8}$ camouflant des milices politiques destinées à briser les grèves et à intimider les militants réformistes, expliquent les violentes convulsions d'une Corée du Sud dont la police n'hésite pas à tirer sur la foule.

Le mécontentement économique et le ressentiment contre la police, coupable de «bavures » répétées, expliquent les conflagrations locales. A Cheju-do, en avril 1948, a éclaté une insurrection ${ }^{9}$ vite récupérée par le Parti du travail du Sud de la Corée ou Nam Chosŏn nodong-dang, émanation du Parti du travail de Pyongyang. Si l'origine de l'affrontement n'est pas directement politique, il le devient ; les renforts de police et les milices formées de réfugiés anticommunistes arrivés de Corée du Nord multiplient les exactions, jetant les mécontents et les insurgés dans les bras du parti du travail. La répression, aveugle, est féroce : l'estimation des pertes humaines varie de 30.000 à 80.000 victimes sur une population de 300.000 habitants environ ${ }^{10}$. The Jeju 4.3 Incident Investigation Report, publié par la Jeju 4.3 Peace Foundation en 2003, a donné 320 morts chez les forces de l'ordre contre 14.028 victimes effectivement recensées. Le comité d'enquête reconnaît cependant qu'une estimation de l'ordre de 25.000-30.000 victimes civiles est correct ${ }^{11}$.

Or, une partie de la gendarmerie coréenne est noyautée par les forces de gauche. Le 20 octobre 1948 , des soldats du $14^{\mathrm{e}}$ régiment, recevant l'ordre de partir pour Cheju-do, se mutinent et sèment la désolation dans la région de Yŏsu-Sunchŏn, avant de se replier sur les monts Chiri pour y créer un maquis. L'apparition de foyers insurectionnels pousse le gouvernement sud-coréen et Yi Sungman à freiner le départ des troupes américaines du pays.

La multiplication des crises, sabotages et insurections pousse le gouvernement à prendre des mesures drastiques. Sous l'émotion de l'affaire de Yŏsu-Sunchŏn, l'Assemblée nationale adopte en novembre 1948 une disposition normative d'exception, dite Loi de Sécurité nationale (Kukka poanpŏp), destinée à châtier les crimes de rebellion, mais aussi de soutien ou d'association à des visées communistes. Comme

\footnotetext{
8 G-2, 27 février 1947, cité par HAN'GUK YŎKSA YŎN'GU-HOE HYŎNDAE-SA YŎN'GU-BAN, Han'guk Hyŏndae-sa 1 : haebang chikhu üi pyŏnhyŏk undong-gwa migunjŏng, Seoul, P'ulpit', 1994, p. 70.

99 Selon les inclinations politiques, on parlera d'insurrection (Cheju-do pallan) ou de résistance populaire (minjung hangjaeng). Les neutralistes parlent simplement «d'affaire» ou "d’incident » de Cheju-do (Cheju-do sakŏn).

10 MERRIL, John, «Cheju-do Ballan», in KAJIMURA, Shûju, Han'guk Hyŏndae-sa yŏn'gu I [Recherches sur l'histoire contemporaine de la Corée I], Séoul, Isŏng gwa hŏnsil-sa, 1988. QUISEFIT, Laurent, Dérapages, exaspération, récupération: l'insurrection de Cheju-do (Corée, avril 1948), in ROUSSEAU Frédéric, SCHMIDT Burckardt (dir.), Les «Dérapages»de la Guerre, XVIe-XXe siècle, Hamburg, DobuVerlag, 2009, pp. 239-257.

${ }^{11}$ The Jeju 4·3 Incident Investigation Report, traduction anglaise, Jeju-si (Jejudo, Corée), 2014. [ 1 ère édition coréenne, décembre 2003].
} 
d'autres dispositions légales prises sous la colonisation, les dispositions de cette Loi de sécurité sont suffisamment vagues pour s'appliquer à une grande variété de crimes de sédition, de sorte qu'en réalité, émus par l'urgence de la situation, les députés coréens ont créé un outil de répression qui peut s'étendre virtuellement à toute forme d'opposition.

L'éradication des guérillas et la guerre larvée sur la frontière sont suivies d'une légère accalmie au début de 1950. La Cordu Nord a en réalité changé de stratégie. Pyongyang attaque le sud le 25 juin 1950, provoquant ainsi la guerre de Corée. Celle-ci est marquée par des atrocités multiples perpétrées par les principaux acteurs militaires, à savoir la Corée du Nord, la Corée du Sud, et les Etats-Unis. Les partisans communistes et l'armée populaire du Nord commirent également leur part d'exactions contre les «ennemis de classe» ${ }^{12}$.

Par la suite, c'est l'armée américaine qui, à plusieurs reprises, décima sciemment certains de ses alliés, et notamment des civils. La révélation en 1999 de l'incident de Nogun-ri, secoua non seulement la Corée du Sud, mais encore les États-Unis. Ces derniers sont probablement responsables, à eux seuls, du décès de 100.000 civils coréens $^{13}$. De 1945 à 1953, on estime en général qu'environ un million de civils sont morts du fait des violences politiques, dont probablement 400.00o en Corée du Sud, sur une population totale de 31 millions d'habitants sur toute la péninsule ${ }^{14}$. Le décompte des victimes de la guerre et des massacres est rendu complexe par les facteurs idéologiques. Le cas de la prison de Taechŏn est exemplaire, puisque les prisonniers communistes y furent exécutés en juillet 1950, au moment de l'avance des Nord-Coréens ${ }^{15}$, et les prisonniers anti-communistes furent abattus par les NordCoréens en retraite après la réussite du débarquement de MacArthur à Inch'ŏn, le 15 septembre $1950^{16}$.

\subsection{Autoritarisme et maintien au pouvoir de Yi Süngman (1952-1960)}

Alors que la guerre de Corée n'est pas encore finie, une grave crise politique agite la vie politique coréenne. La constitution de 1948 interdisait expressément un second

\footnotetext{
${ }^{12}$ REES, David, Korea, the Limited War, New York, Saint Martin Press, 1964, pp. 93-94.

${ }_{13}$ En novembre 1999, Le Star and Stripes, journal des Armées des Etats-Unis, indiquait que la Corée du Nord imputait aux États-Unis la mort de 148.000 personnes, mais ces chiffres comprennent les victimes des bombardements sur les villes et les complexes industriels de la Corée du Nord.

14 Dont environ 20 millions au Sud.

${ }^{15}$ DEANE, Philip, I Was A Captive in Korea, New York, W.W. Norton \& Company, 1953, p. 85.

16 Rapport de mission du capitaine Monteagle, MINISTÈRE DES AFFAIRES ETRANGÈRES

(France), Asie Océanie, Corée, carton 1, f. 227 sq.
} 
mandat au président, élu par l'Assemblée nationale ${ }^{17}$. Dès le 30 mai 1950 Yi Sŭngman avait perdu la majorité au parlement. Les partis étaient largement désavoués, arrivant après les députés indépendants, et le parti du président en quatrième position. Or, le président était élu pour quatre ans et ce désaveu fut provisoirement oublié du fait de l'éclatement de la guerre, moins d'un mois plus tard.

Le gouvernement déposa à l'Assemblée en novembre 1951 un projet de révision de la Constitution prévoyant l'élection du président de la République au suffrage universel direct, ainsi que la création d'un système parlementaire bicaméral, pensé pour diluer la force du pouvoir législatif. Les deux principaux partis, les Démocrates Nationalistes et les Libéraux, s'opposèrent à ce projet, considéré comme mal rédigé, puisque le texte ne prévoyait pas la responsabilité du gouvernement devant l'Assemblée, ce qui nuisait à l'équilibre des pouvoirs.

En janvier 1952, l'Assemblée nationale rejeta par un vote écrasant la proposition de réforme constitutionnelle (19 voix pour, 143 contre, 1 abstention ${ }^{18}$ ), humiliant ainsi profondément le vieux président.

En avril 1952, un nouveau projet d'amendement de la Constitution, prévoyant l'adoption d'un régime parlementaire, et revêtu de la signature de 124 députés, fut déposé. Le nombre de députés signataires représentait une voix de plus que le minimum nécessaire à l'adoption de cette réforme. En mai, ces députés déposèrent un nouveau projet, légèrement modifié, après le rejet de l'amendement par le gouvernement. La tension entre les pouvoirs exécutif et législatif provoqua une crise grave, connue comme la « crise politique de Pusan », Pusan chŏngch`i p’adong.

À Pusan ${ }^{19}$, une grande manifestation organisée avec soin, patronnée par plusieurs associations patriotiques proches du Parti libéral de Yi Sŭngman, réunit le 19 mai 1952, de 1.000 à 1.500 personnes $^{20}$. La foule marcha sur l'Assemblée qui était en session. Les forces de l'ordre, toutefois, empêchèrent toute violence ${ }^{21}$. Tout le mois de mai, des manifestations violentes furent organisées pour faire pression sur les députés. La loi martiale fut promulguée le 25 mai, dans la région de Pusan et 44 députés furent arrêtés $^{22}$ et détenus par la police militaire ${ }^{23}$. Pourtant, le 28, l'Assemblée, bien que diminuée, vota à 96 voix contre 3 l'abolition de la loi martiale. Les observateurs de

\footnotetext{
${ }_{17}$ Article 55 de la Constitution de 1948.

${ }^{18}$ A noter aussi que tous les députés n'étaient pas présents, du fait de la guerre.

19 Pusan fut la capitale du temps de guerre.

${ }^{20}$ Le chiffre est relativement important, alors que les forces vives du pays sont mobilisées par la guerre.

${ }_{21}$ Rapport de la CIA, Daily Korean Bulletin, OCI 6012, 21 May 1952.

${ }^{22}$ L'arrestation et la détention des députés est pourtant illégale selon la Constitution.

23 CIA, Korea Daily Bulletin, OCI 6017, 27 May 1952.
} 
l'ONU prônèrent la levée de la loi martiale et la libération des députés ${ }^{24}$. Le 12 juin, 12.00o personnes vinrent à Pusan pour soutenir le président Yi Sŭngman, conspuant une Assemblée corrompue et déloyale ${ }^{25}$. Associations féminines, groupes de la jeunesse, étudiants, employés de sociétés contrôlées par l'Etat, aidés dans leurs déplacements par la mise à disposition de véhicules par la présidence, vinrent soutenir le président, réclamer la dissolution de l'Assemblée, et dénoncer les interférences étrangères. Les discours enflammés n'agitèrent pourtant pas la foule, passive et disciplinée ${ }^{26}$. Face à cette pression du pouvoir présidentiel, le premier ministre lui-même se réfugia sur un navire-hôpital américain.

C'est sans surprise que le 7 juillet, la Constitution fut révisée, principalement les articles 53 et 54 concernant les dispositions relatives au mandat du président. Le principe de l'élection du président au suffrage universel direct à bulletin secret était désormais établi ${ }^{27}$. Aussi est-ce sans surprise que le 5 août 1952, Yi Sŭngman fut élu président au suffrage universel, à une écrasante majorité, 74\% des votes, contre 11,4 \% pour l'indépendant Cho Pong-am ${ }^{28}$ et $10,9 \%$ pour le parti démocratique national de Yi Si-yŏng29.

Pourtant, si Yi Sŭngman avait fait taire la fronde des députés, il avait beaucoup appris du flottement politique, et n'était pas satisfait. En 1954, il parvint à nouveau à faire amender la constitution, afin de pouvoir se représenter aux élections ${ }^{30}$. Yi Sŭngman et ses partisans eurent alors recours à un stratagème pour arrondir au nombre supérieur, de façon artificielle, le pourcentage des suffrages obtenus. La Constitution ainsi adoptée de force, Yi Sŭngman fut élu président de la République pour la troisième fois le 15 mai 1956, avec 70 \% des suffrages exprimés.

Dès lors, Yi Sŭngman se montra despotique: ne supportant plus la moindre opposition à son encontre, ni même à l'égard de son gouvernement, il ordonna en avril 1959 l'arrêt de la publication d'un quotidien national d'opposition, le « Kyŏnghyang shinmun » ou Journal de la Capitale et des Provinces. En juillet 1959, le président fit même exécuter Cho Pong-am (1898-1959), son ancien rival à l'élection présidentielle, sous un fallacieux prétexte d'espionnage au profit de la Corée du Nord, aidé en cela par

\footnotetext{
24 CIA, Korea Daily Bulletin, OCI 6019, 29 May 1952.

25 CIA, Korea Daily Bulletin, OCI 4030, 12 June 1952.

${ }^{26}$ CIA, Korea Daily Bulletin, OCI 6032, 14 June 1952.

27 Voir SŎNG, Nak-in, Taehan min'guk hŏnbŏp-sa [Histoire de la Constitution de la République de Corée], P’aju, Pŏmmun-sa, 2012, p. 404-405.

28 Cho Bong-Am fut par la suite condamné pour activités communistes et exécuté.

29 Ce dernier avait un temps été le vice-président de Yi Sŭngman.

30 Voir la Constitution de 1954, notamment les articles 52 et suivants.
} 
la loi de sécurité nationale (Kukka poanbŏp) qui semble avoir été dévoyée pour la première fois à un si haut niveau, à des fins de vile politique.

\subsection{Chute de Yi Sŭngman}

Homme du XIXe siècle, éduqué par des missionnaires et ayant passé une partie de sa vie en exil aux Etats-Unis ${ }^{31}$, Yi Sŭngman comprend mal les mutations du pays qu'il dirige. Plutôt que de réformer ou de quitter le pouvoir, il durcit ses positions.

Incapable d'abandonner le pouvoir, Yi Sŭngman se présenta une quatrième fois à l'élection présidentielle, le 15 mars 1960. Il aurait cette fois pu être élu sans difficulté, du fait du décès prématuré de son unique adversaire, Cho Pyŏng-ok (1894-1960), mort de maladie. Les partisans du président organisèrent pourtant des fraudes électorales afin de permettre à Yi Ki-pung (1896-1960), alors président de l'Assemblée nationale, d'être élu en tant que vice-président. Le Parti libéral au pouvoir utilisa tous les moyens légaux et illégaux à sa disposition pour obtenir la majorité, et obtint finalement $87 \%$ des voix, sans que personne ne soit dupe.

Dès le 28 février, les étudiants de Taegu commencèrent à manifester contre les fraudes manifestes qui émaillaient la campagne électorale. Le 15 mars, jour des élections, les étudiants de Masan manifestèrent à leur tour en masse pour demander l'annulation de l'élection de Yi Ki-pung. La police, appliquant des procédures récurrentes, aux conséquences le plus souvent funestes, tira dans la foule. Il y eu des morts et des blessés. Le 11 avril, on découvrit sur une plage de Masan le corps sans vie d'un lycéen, une grenade lacrymogène enfoncée dans le crâne ${ }^{32}$, preuve que la police avait tiré directement sur les manifestants, et non au-dessus de la foule.

L'indignation fut unanime ; le mouvement se radicalisa, tournant à l'émeute. Le 19 avril, la police tira à nouveau dans la foule rassemblée devant le palais présidentiel 33 : on releva cette fois 142 morts, preuve d'une flagrante incompétence de la police autant que du mépris du pouvoir envers la population.

${ }^{31}$ Il ne rentre en Corée qu'en 1945, après un long exil aux Etats-Unis et en Chine.

${ }^{32}$ KIM C. I. Eugene \& KIM Ke-soo, "The April 1960 Korean Student Movement", The Western Political Quarterly, Vol. 17, No. 1 (Mar., 1964), p. 85.

33 Ancien immeuble du gouvernorat japonais, ce bâtiment a été détruit en 1995. 
Tab. 1. Victimes de la Révolution d'Avril (Avril 1960).

\begin{tabular}{|l|l|l|}
\hline Catégories & Victimes (martyrs) & $\mathbf{\%}$ \\
\hline Collégiens & 19 & 10,2 \\
\hline Lycéens & 36 & 19,4 \\
\hline Etudiants & 22 & 11,8 \\
\hline Employés & 10 & 5,4 \\
\hline Ouvriers & 61 & 32,8 \\
\hline Chômeurs & 33 & 17,7 \\
\hline Autres & 5 & 2,7 \\
\hline Total & 186 & 100.00 \\
\hline
\end{tabular}

Source : YI Ch'ang-ŏn34, 1960 nyeondae 4 wŏl hyŏngmyŏng - P'ogi hal su omnŭn kach’i, minjujuŭi [La révolution d'avril 1960 : jusqu'à ce qu'on ne puisse renoncer à la démocratie]. (Cours du 20 octobre 2010, Korea University).

Ce massacre ouvrit un cycle de protestations. Le lendemain, la foule des manifestants, soutenue par la majorité de la population, se retourna contre Yi Sŭngman, alors âgé de 85 ans. Le cycle d'action et de répression qui s'était exaspéré du fait des bavures policières, emporta finalement le régime le 26 avril. Humilié, effrayé par ce désaveu populaire et par la dissolution du lien entre la société et ses dirigeants, Yi Ki-pung se suicida avec sa famille le 24 avril. Vieilli, dépassé par ce mouvement populaire qu'il comprenait mal, Yi Sŭngman fut contraint à la démission le 26 avril. S'exilant en mai à Hawaii, il y mourut en juillet 1965.

\subsection{Transition démocratique infructueuse}

Le flottement politique et institutionnel consécutif au départ de Yi Sŭngman, avait déclenché une détestation générale envers l'ancien «père de la patrie ». Le nouveau régime, parlementaire, s'était enlisé dans des querelles politiques stériles, marquées non seulement par des divergences de vues, mais encore par des cissions en cascades au sein des partis. La stabilité politique ne fut jamais réalisée, le gouvernement du Premier ministre John Chang (Chang Myŏn, 1899-1960), donnant l'impression d'être indécis, trop honnête, et de manquer de courage, traits de caractères incompatibles avec ceux d'un véritable dirigeant.

En outre, la presse, libérée de toute censure, considérait avec suspicion tout mouvement du nouveau gouvernement, dans un contexte marqué par un extrême désir de changement assorti de sévères critiques envers des politiques souvent issus du régime précédent.

34 Professeur, département de sociologie, Korea University (Koryŏ Taehkkyo), Séoul. 
Aucune personnalité isssue de la révolution d'avril n'entra au gouvernement, et aucun parti réformateur n'obtint une influence significative dans le processus électoral. Aux élections générales de juillet, seuls quatre sièges furent remportés par ces partis à la Chambre des représentants, et trois à la Chambre des Conseillers. Aussi, ces partis, marginalisés, eurent-ils recours aux défilés de protestation pour exprimer leur désir de changement.

En quelques mois, Chang Myŏn remania à quatre reprises son cabinet. Le pays se trouvait maintenant dans une situation économique catastrophique. En outre, les étudiants, fiers de leur rôle déterminant dans la chute de l'ancien régime se mêlaient désormais de tout et manifestaient pour les motifs les plus ridicules et les moins clairs, n'hésitant pas à envahir l'Assemblée nationaler pour demander l'écartement définitif des postes à responsabilité des anciens soutiens de Yi Sŭngman (11 octobre). Le jour suivant, l'Assemblée vota une loi autorisant le passage en jugement de personnes précédemment acquitées, notamment en matière de brutalités policières. La police, tout en accompagnant et en canalisant certains mouvements de foule, ne fit rien pour s'opposer à ces débordements, malgré la sauvagerie de l'émeute.

Désordres, critiques, vélléités, manque de réactivité, le gouvernement semblait laisser faire, l'Assemblée changeait de vues sous la pression de la rue et votait les lois en conséquence. L'anarchie semblait sur le point de régner en maître.

\subsection{La dictature militaire}

Dans la lourde atmosphère de 1960-1961, faite de rumeurs, de défilés et d'actions étudiantes virulentes, la nécessaire acceptation du jeu politique semblait faire défaut. Le gouvernement semblait balloté, bousculé par les actions des forces militantes, au point que, pour certains Coréens, le système démocratique lui-même devint un problème, puisqu'il s'avérait incapable de décisions franches et de stabilité. Pour les étudiants et les intellectuels, la démocratie n'allait pas assez loin. Pour d'autres, et notamment pour les élites urbaines et certaines autorités, elle constituait même, en l'était des choses, une menace pour l'ordre social35. En outre, le prix du riz avait augmenté de 60\% entre décembre 1960 et avril 1961 tandis que le chômage dépassait

35 ECKERT, Carter J.J., LEE, Ki-baik, LEW, Young-ick et al., Korea, Old and New: a History, Seoul, Ilchokak \& Cambridge Mass., Harvard Univ. Press, 1990, p. 358. 
les $23 \%$. Non seulement la vie politique était chaotique, mais encore des pénuries alimentaires étaient apparues ${ }^{36}$.

Le 16 mai 1961, le gouvernement Chang Myŏn fut renversé par un coup d'Etat militaire37. Les putschistes regroupés au sein d'un Comité militaire révolutionnaire (CMR), étaient théoriquement dirigés par le chef d'Etat-Major de l'Armée, le général Chang Do-yong. En réalité, c'est le général Park Chung-hee (Pak Chŏng-hŭi) qui s’imposa comme le nouvel homme fort du régime ${ }^{38}$.

\subsection{Installation et transformations}

Le 20 mai, le CMR devint le Conseil Suprême pour la Reconstruction Nationale (CSRN). Celui-ci s'arrogea tous les pouvoirs, supervisant le fonctionnement de toute l'administration et promulguant les lois ${ }^{39}$. Le CSRN décida la dissolution de l'Assemblée nationale, mettant ainsi fin à l'éphémère $\mathrm{II}^{\mathrm{e}}$ République. Des milliers de fonctionnaires corrompus furent suspendus, et des centaines de politiciens interdits d'activité politique. Les organisations violentes associées à la pègre et rémunérées par les hommes politiques comme hommes de main furent démantelées, les voyous jetés en maisons de rééducation. Les réseaux de prostution furent détruits. Des centaines de dancings, bars et mêmes salons de thé40 durent fermer ${ }^{41}$.

Dès juin 1961, le CSRN fonda la KCIA (Korean CIA), agence cumulant le renseignement extérieur et la sûreté intérieure, autorisée à contrôler les autres agences de sécurité, et bientôt appelé à jouer le rôle de police politique. En trois ans, la KCIA avait établi un vaste réseau d'agents en Corée et à l'étranger.

Le nouveau régime se positionna dans un anticommunisme strict, propre à lui assurer le soutien des États-Unis en pleine Guerre Froide, bien qu'en pratique, ses opinions soient plus souples que celles de Yi Sŭngman.

Le 12 août, le SCRN annonça qu'il rendrait le pouvoir aux civils en 1963, après un référendum et l'adoption d'une nouvelle Constitution. Le général Park prononça

\footnotetext{
${ }^{36} \mathrm{KIM}$, Hyung-A, Korea's Development Under Park Chung Hee: Rapid Industrialization, 196179, London-New York, Routledge-Curzon, 2004.

37 On parle en coréen de O il-lyuk gunsa jŏngbyŏn « coup d'Etat du 16 mai ». Jusqu'en 1993, le discours officiel parlait d'une « révolution du 16 mai » (O il yuk hyŏngmyŏng).

${ }^{8} 8$ Chang Do-yong sera arrêté en juillet suivant.

39 KIM, Byung-Kook, VOGEL, Ezra F. (dir.), The Park Chung Hee Era: The Transformation of South Korea, Cambridge (Mass.), Harvard University Press 2011, p. 88.

40 Tabang ; c'est l'équivalent coréen du « café ».

${ }^{41}$ ECKERT, Carter J.J., LEE, Ki-baik, LEW, Young-ick et al., op. cit., p. 361.
} 
bientôt la relaxe des anciens leaders politiques, ce qui lui permit d'être invité par le président Kennedy à Washington en novembre 1961.

En décembre 1962, la loi martiale fut levée et le résultat du référendum autorisa la fondation de la III ${ }^{e}$ République, dans laquelle le président devait être élu au suffrage universel direct. En janvier 1963, une activité politique étroitement encadrée fut à nouveau autorisée, de manière à permettre la tenue d'élections législatives et présidentielles la même année. Pourtant, dès 1962, 4000 anciens politiques avaient été exclus de toute activité publique, écartant ainsi de la compétition les plus éminents rivaux. En décembre, le général Park annonça son intention de quitter l'armée pour se présenter aux élections, et que les autres membres du SCRN étaient libres de l'imiter.

Les élections présidentielles du 15 octobre et les législatives de novembre 1963 permirent la formation d'un gouvernement «civil». Le 17 décembre 1963, la III République naquit officiellement en tant que fille du putsch de 1961. La junte affirmait avoir renforcé le leadership démocratique et assuré la survie de la République pendant la crise. L'expérience du passé avait mené à l'installation d'un exécutif fort, avec un renforcement de la responsabilité politique des politiciens ${ }^{42}$.

La nécessité de fournir un remède constitutionnel à l'inefficacité du Parlement, provoqua ainsi un retour flagrant à un système qui avait déjà généré une dictature personnelle, sous le président Yi Sŭngman43.

\subsection{Ajustements politiques}

En 1972, le système avait largement montré ses limites. Le Parti Démocratique républicain (PDR) ${ }^{44}$, majoritaire au Parlement, avait dû siéger secrètement pour faire passer un amendement permettant au président sortant de se représenter à un troisième mandat en 1971. Au début des années 1970, l'opposition avait réussi à s'organiser, au sein du Nouveau Parti Démocratique (NPD), autour de figures telles que Yu Chin-san, Kim Young-sam et Kim Dae-jung.

Les élections présidentielles de 1971 illustrèrent le grave effritement de la position du parti présidentiel. Kim Dae-jung, le jeune candidat du NPD, remporta $45 \%$ des votes, devançant même l'ancien président, Yun Po-sŏn. Si Park Chung-hee conservait le pouvoir, sa situation devenait difficile, d'autant que Kim Dae-jung avait remporté le vote des villes (52\%), et même raflé la mise à Séoul (60\%). Le parti présidentiel (PDR)

42 PAK, Chi Young, «The Third Republic Constitution of Korea: An Analysis», in Western Political Quarterly, 21, 1/1968, pp. 110-122, p. 113.

43 Ibidem.

44 Minju konghwa-dang. 
perdait 16 sièges, et avec eux, la majorité des deux tiers à l'Assemblée, mais se maintenait grâce au vote rural. Le NPD, incapable d'exploiter sa victoire, se perdit pourtant dans les joies amères du factionalisme.

Menacé dans sa position, le pouvoir réagit promptement. Prétextant des menaces intérieures et extérieures45, Park décréta l'état d'urgence en décembre 1971. Dix mois plus tard (octobre 1972), il instaura la loi martiale. La Constitution fut à nouveau suspendue, l'Assemblée nationale dissoute, de même que les partis politiques. Toute activité politique fut interdite, les libertés civiles restreintes, notamment la liberté de parole.

La nouvelle constitution, dite Constitution Yusin (« revitalisation »), fut adoptée par un référendum auquel les citoyens, intimidés, accordèrent leur «confiance » en novembre 1972. Cette Constitution transforma la présidence en « dictature légale »46.

Le régime devint plus autoritaire non seulement du fait de la pression de l'opposition, mais aussi en raison des conflits entre le pouvoir en place et une société aspirant à plus de liberté et de confort. Il est vrai que les relocalisations forcées (destruction de certains quartiers au nom de la modernisation), les protestations contre les impôts, les atteintes à la société traditionnelle pouvaient alimenter les mécontentements. En août 1971, environ 30 ooo personnes des banlieues de Séoul se révoltèrent contre le gouvernement réclamant des exemptions d'impôts et des occasions d'emploi, en même temps que les syndicats se radicalisaient ${ }^{47}$.

Le régime se durcit aussi en raison des manœuvres de la Corée du Nord ou de ses affidés, notamment après la mort, en 1974, de Yuk Yŏng-su, épouse du président, tuée dans un attentat visant son mari. Cet assassinat entraîna des mesures sécuritaires drastiques, qui contribuèrent à isoler le président, même si le décès de la présidente, fort aimée, procura un temps quelques sympathies populaires au malheureux époux.

\subsection{Répressions}

Les gouvernements de Park Chung-hee usèrent de tous les moyens de coercition possibles, pour conserver le pouvoir. Les opposants, politiques ou littéraires, furent pourchassés, souvent sous des prétextes fallacieux, et incarcérés, parfois pour peu de temps, parfois pour des peines plus longues. La menace nord-coréenne, par ailleurs réelle, fut invoquée à plusieurs reprises, pour s'assurer de la personne des opposants

45 Les incidents de frontière avec le nord sont à cette époque très nombreux. ${ }^{46}$ ECKERT, Carter J.J., LEE, Ki-baik, LEW, Young-ick, et al., op. cit., p. 365.

47 IM, Hyug Baeg, "The Rise of Bureaucratic Authoritarianism in South Korea», in World Politics, 39, 2/1987, pp. 231-257, p. 255. 
politiques, à travers l'application de la Loi de Sécurité nationale ${ }^{48}$. Dans cette lutte contre les oppositions, la KCIA joua un rôle crucial, y compris lors d'opérations à l'étranger, comme le kidnapping de Kim Dae Jung au Japon en 1973. De même, le poète populaire Kim Chi Ha fut incarcéré dans les années 1970 pour ses poèmes critiquant la société, notamment Rumeurs sans fondements, ou Cinq bandits, et fut accusé de communisme, ce qui pouvait lui valoir la corde. Or, il était catholique. Il réclamait la liberté d'écriture, donc de pensée. Son cas émut la communauté intellectuelle et politique internationale, de sorte que de nombreuses personalités signèrent une pétition en sa faveur, notamment Jean-Paul Sartre, Simone de Beauvoir, Noam Chomsky, Edwin Reischauer, ou encore le chancelier Willy Brandt 49 .

Le professeur Paik Nak-chung50 fut lui brièvement interrogé par la KCIA après avoir critiqué la mise en détention du romancier Nam Chong-hyŏn${ }^{51}$, auteur du roman antiaméricain bunji (196552), qui contenait de sérieuses critiques du système Park chunghee. De telles détentions, ou « accompagnements volontaires », selon la dénomination officielle, devinrent encore plus fréquents après le « second coup d'Etat » de 1972, qui garantit la présidence à vie au général-président. En 1974, Paik Nak-chung fut chassé de son poste universitaire par le Ministère de l'Education, au motif qu'il avait signé une pétition réclamant une constitution démocratique. En 1977-1978, Paik Nak-chung fut aussi jugé pour publication de livre «pro-communiste». Or, il s'agissait de la traduction de divers rapports sur la Chine publiés par des journalistes et des chercheurs japonais et occidentaux, compilés par le professeur Yi Yŏng-hŭi (1929-2010)53. La maison d'édition Changbi, et son journal trimestriel Création et Critique, entrèrent dans une phase de vicissitudes marquée par la censure, la confiscation des textes publiés, et l'emprisonnement des contributeurs, tels que Yi Yŏng-hŭi ou les poètes Kim Chi-ha et Ko Un54. A noter pourtant que la revue littéraire ne fut jamais interdite de parution, les numéros incriminés étant simplement confisqués.

Sur le plan politique, la figure la plus éminente de l'opposition fut sans doute Kim Dae-Jung (1925-2009), qui fut maintes fois arrêté par le pouvoir. En août 1973, il fut

\footnotetext{
${ }^{48}$ KRAFT, Diane, «South Korea's National Security Law: A Tool of Oppression in an Insecure World», in Wisconsin International Law Journal, 24, 2/2006, pp. 627-658.

49 New York Times Magazine, January 7, 1973.

$5^{\circ}$ Professeur de littérature anglaise à l'Université nationale de Séoul.

${ }^{51}$ Ecrivain dont les critiques sociales acerbes contre le régime lui valurent plusieurs peines d'emprisonnement.

$5^{2}$ La Corée y est présentée comme un pays pollué par la corruption et les abus de pouvoir, ainsi que par l'anti-américanisme. L'œuvre fut reprise par un journal nord-coréen, donnant un prétexte de plus pour accuser l'auteur de sympathies communistes.

53 Journaliste et professeur d'université.

54 Célèbre poète engagé coréen.
} 
même enlevé à Tôkyô par des agents de la KCIA et reconduit en Corée, où il fut à nouveau incarcéré.

Pourtant, au-delà de ces cas emblématiques55, les chiffres sont éloquents : de 1961 à 1979, plusieurs milliers de politiques et d'intellectuels, de syndicalistes et d'opposants, ont été arrêtés en raison des lois édictées par les différents régimes politiques nés sous la houlette du président-général Park. Environ quatre mille politiques et fonctionnaires corrompus ont été arrêtés au lendemain du coup d'Etat, puis généralement relâchés. La loi de Sécurité nationale a conduit 1968 personnes en prison. Celle contre les activités communistes 4172, et la loi sur la « sécurité sociale » sahoe anjŏn pŏp, condamnant des comportements "anti-sociaux », 19 personnes, ce qui donne un total d'environ dix mille personnes ${ }^{5}$. En dépit de quelques condamnations à la peine capitale, la majorité des personnes incarcérées furent libérées au bout d'un certain nombre d'années de prison.

Tab. 2. Arrestations au titre des différentes lois en vigueur sous Park Chung-hee.

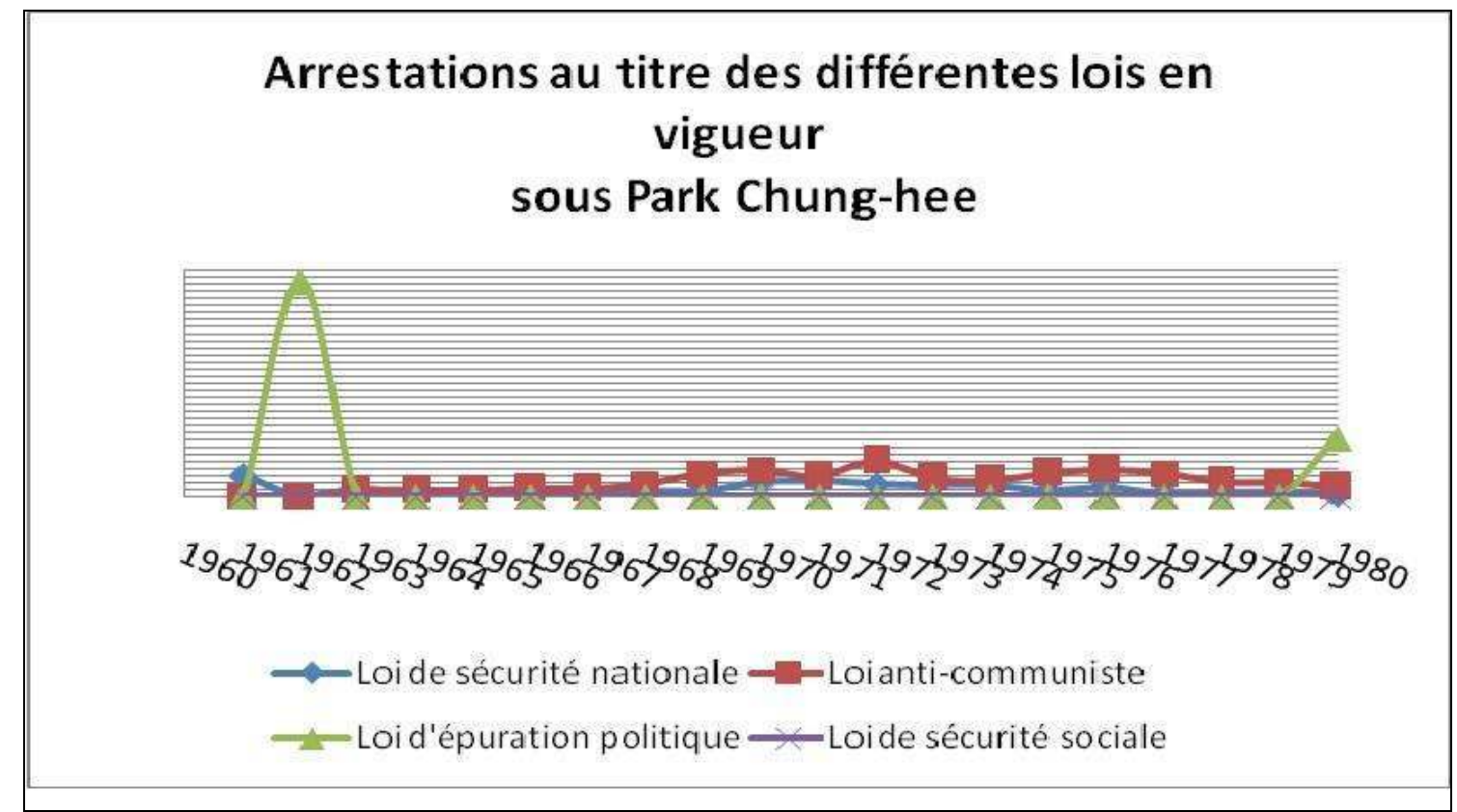

Source: Établi par nos soins, d'après PAK Chong- sŏn, Han'guk chŏngch’i wa chŏngch’i pongnyŏk, Seoul, Seoul Taehakkyo ch'ulp'anbu, 2001.

55 Voir PAK Chong-sŏn, Han'guk chŏngch’i wa chŏngchii pongnyŏk (la politique coréenne et la violence politique), Seoul Taehakkyo ch'ulp'anbu, 2001.

${ }^{56}$ Non compris les condamnations liées aux conflits du travail, qui apellent une étude séparée. 
Tab. 3. Tableau récapitulatif des principales victimes des répressions sous Park Chung-hee (1961-1979)

\begin{tabular}{|l|l|l|l|l|l|}
\hline $\begin{array}{l}\text { Dispositions } \\
\text { normatives }\end{array}$ & $\begin{array}{l}\text { Loi de } \\
\text { sécurité } \\
\text { nationale }^{1}\end{array}$ & $\begin{array}{l}\text { Loi anti- } \\
\text { communiste }\end{array}$ & $\begin{array}{l}\text { Loi } \\
\text { d'épuration } \text { politique }^{3}\end{array}$ & $\begin{array}{l}\text { Loi de } \\
\text { sécurité } \\
\text { sociale4 }\end{array}$ & $\begin{array}{l}\text { Total } \\
\text { général }\end{array}$ \\
\hline $\begin{array}{l}\text { Nombre de } \\
\text { personnes } \\
\text { arrêtées }\end{array}$ & 1.968 & 4.172 & 3.849 & 19 & 10.008 \\
\hline
\end{tabular}

Source: PAK Chong-sŏn, Han'guk chŏngch`i wa chŏngch`i pongnyok, Seoul, Seoul Taehakkyo ch'ulp’anbu, 2001.

${ }^{1}$ Kukka poanpŏp, instaurée en décembre 1948.

${ }^{2}$ Pankongpŏp, promulguée en 1961.

3 Chŏngch’i hwaldong chonghwapŏp établie en 1961 contre les politiciens.

4 sahoe anjŏn pŏp, instaurée en 1975.

Malgré les arrestations fréquentes des opposants, l'élimination physique n'a pas été de mise et les peines prononcées contre les opposants, souvent commuées. Le 22 mars 1977, la décision de la Cour suprême de Corée annula cinq condamnations sur réquisition du ministère public, les autres opposants prévenus étant condamnés à des peines de 1 à trois ans de prison, parfois avec sursis.

On est donc loin des exécutions systématiques et souvent sans jugement, de l'époque de Yi Sŭngman, même si l'usage de la torture a continué. Le contexte d'urgence nationale et d'affaiblissement politique de la guerre et de l'avant-guerre, explique en partie les atrocités sous Yi Sŭngman, de même que l'émergence des nouvelles techniques de maintien de l'ordre, comme les gaz lacrymogènes, peut expliquer la baisse du nombre des victimes.

Tab. 4. Nombre d'arrestation : Loi de sécurite nationale et loi anticommuniste (1960-1980).

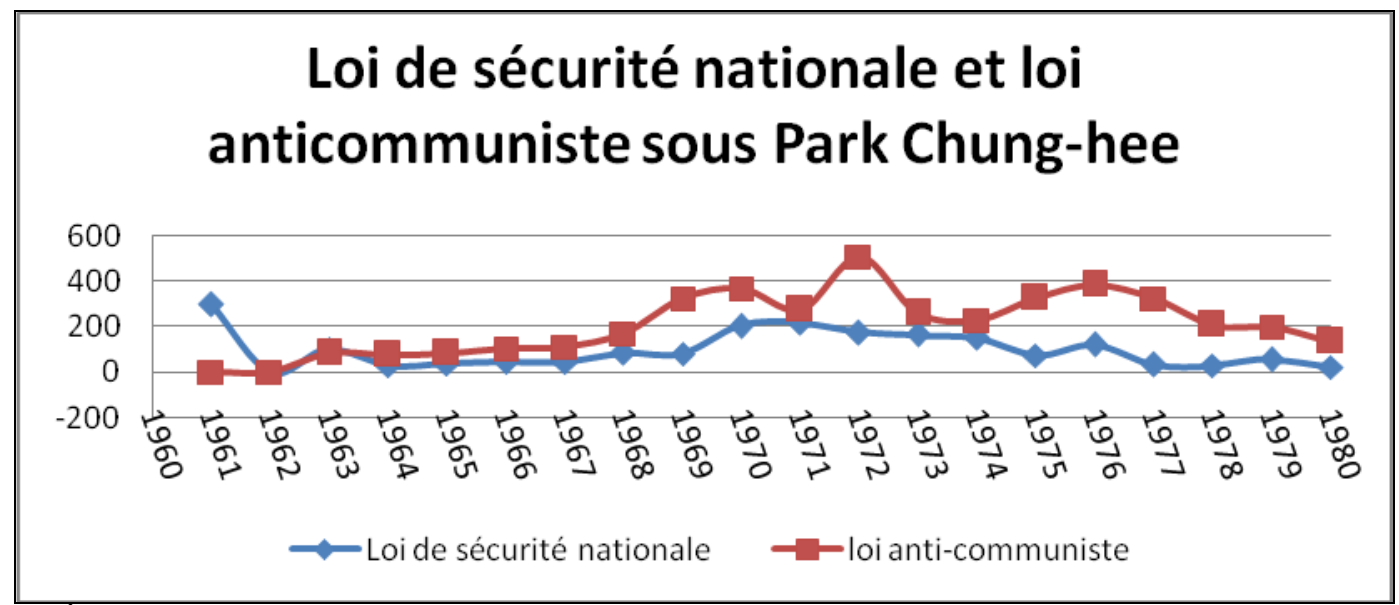

Source: Établi par nos soins d'après PAK Chong-sŏn, Han'guk chŏngch'i wa chŏngch'i pongnyok, Seoul, Seoul Taehakkyo ch'ulp'anbu, 2001. 


\section{Politiques économiques}

Un autre volet du dossier concerne les politiques économiques de Yi Sŭngman et de Park Chung-hee. Yi se contenta toujours de déclarations générales, sans disposer d'une vision économique claire, de sorte qu'il ne mena jamais de politique économique proprement dite, et ne fit que soutenir des initiatives privées, selon un système qui permettait surtout le financement de son parti et la transformation de l'aide économique américaine en manne pour son parti. Au contraire, plus conscient des enjeux, Park et ses gouvernements s'ingénièrent à développer l'économie dans une perspective volontariste, destinée non seulement à fonder les bases de la croissance, mais surtout capable d'asseoir une véritable puissance industrielle et d'assurer sa pérennité.

Entre Park Chung-hee et Yi Sŭngman, on observe un saut de génération. Yi Sŭngman reste un homme du XIXe siècle, qui comprend mal les mécanismes complexes de l'économie moderne. Au contraire, Park Chung-hee, par sa formation d'instituteur et d'officier, a compris les enjeux économiques et financiers du monde moderne.

Si la guerre ruina totalement la Corée, détruisant l'essentiel de ses villes et de son industrie, la reconstruction fut relativement lente. Pourtant, les États-Unis d'une part, divers organismes onusiens d'autre part, fournirent des subsides importants à la Corée du Sud, en vue de son redressement.

Cependant, l'observation de l'évolution du PNB par habitant, quand bien même elle ne donne que des données artificielles et simplistes - le coût réel de la vie n’y entre pas en ligne de compte - offre des perspectives intéressantes. Les fluctuations économiques sont encore corrélées, à cette époque, à des facteurs complexes qui prennent en compte notamment la qualité des récoltes, la société restant encore largement rurale. Cependant, malgré un taux de croissance relativement élevé, (5,1\% en 1954 puis 4,5 en 1955) l'économie reste fragile, et connaît un profond recul en 1956, avec une croissance négative (-1,4\%). Le bond significatif de 1957 montre un effet de récupération. Si la résilience d'après-guerre est en marche, le système semble s'essoufler rapidement, avec $3,8 \%$ à peine en 1959. Il est vrai également que le mauvais chiffre de 1960 peut être imputé en partie aux grèves et mouvements sociaux qui vont provoquer la chute du régime de Yi. 
Tab. 5. Evolution du PNB par habitant, 1953-1960.

\begin{tabular}{|l|l|l|}
\hline Années & PNB/habitant (en US\$) & Taux de croissance (\%) \\
\hline 1953 & 67 & \\
\hline 1954 & 70 & 5,1 \\
\hline 1955 & 65 & 4,5 \\
\hline 1956 & 66 & $-1,4$ \\
\hline 1957 & 74 & 7,6 \\
\hline 1958 & 80 & 5,5 \\
\hline 1959 & 81 & 3,8 \\
\hline 1960 & 79 & 1,1 \\
\hline
\end{tabular}

Source: Etabli par nos soins d'après : BANK OF KOREA, Kyeongje t’ongge nyeonbo, cité par HO John KieChang, Korean Politics : the Quest for Democratization and Economic Development, Ithaca \& London, Cornell University Press, 1999, p. 62.

La majorité de la population reste d'autant plus pauvre que l'inflation, certes commencée pendant la guerre, n’est pas endiguée, bien au contraire. Non seulement la masse monétaire en circulation augmente, mais les prix à la consommation augmentent eux aussi de manière significative.

Yi Sŭngman ne dispose pas des fonds nécessaires pour une politique de développement. Ses compétences en économie sont d'ailleurs très réduites. Après avoir augmenté la masse monétaire en circulation, il cède soudain aux pressions américaines et tente d'inverser la tendance en faisant mettre les scellés sur les presses le 11 février 1954. Le président de la Banque de Corée les fera ôter, expliquant au président qu'un tel arrêt bloquerait toutes les opérations monétaires du gouvernement. Les presses se remirent à tourner, aggravant l'inflation.

En réalité, aucun programme de développement économique réel ne fut organisé par Yi Sŭngman. Seuls de grands discours furent formulés, se contentant de généralités, quand bien même ils pouvaient être marqués parfois d'un bon sens simpliste. Ce n'est qu'en 1957 qu'un programme de développement de trois ans fut amorcé par le ministère de la Recontruction. Pourtant, il ne fut approuvé que le 15 avril 1960, à la veille du mouvement étudiant. La principale contribution de Yi Sŭngman à l'économie fut de vanter et de mettre en lumière l'ardent besoin d'aide économique et militaire dont le pays avait besoin, afin de maximiser l'obtention des subsides étrangers. Il se fit aussi l'avocat d'importations capables de remplacer les produits insuffisamment fabriqués en Corée, ce qui aggrava la situation.

En outre, l'aide américaine servait d'abord à soutenir l'industrie américaine, avec des budgets alloués à des achats d'armes destinés à moderniser l'armée sud-coréenne. De plus, l'aide américaine en nature (riz, farine), était revendue à la population, au 
profit du gouvernement et au détriment des paysans. Les aides destinées au financement des entreprises étaient souvent détournées, au profit des caciques du régime et du financement du parti de Yi Sŭngman.

Tab. 6. Circulation de l'aide américaine, des ressources économiques et des fonds monétaires sous le gouvernement de Yi Seungman.

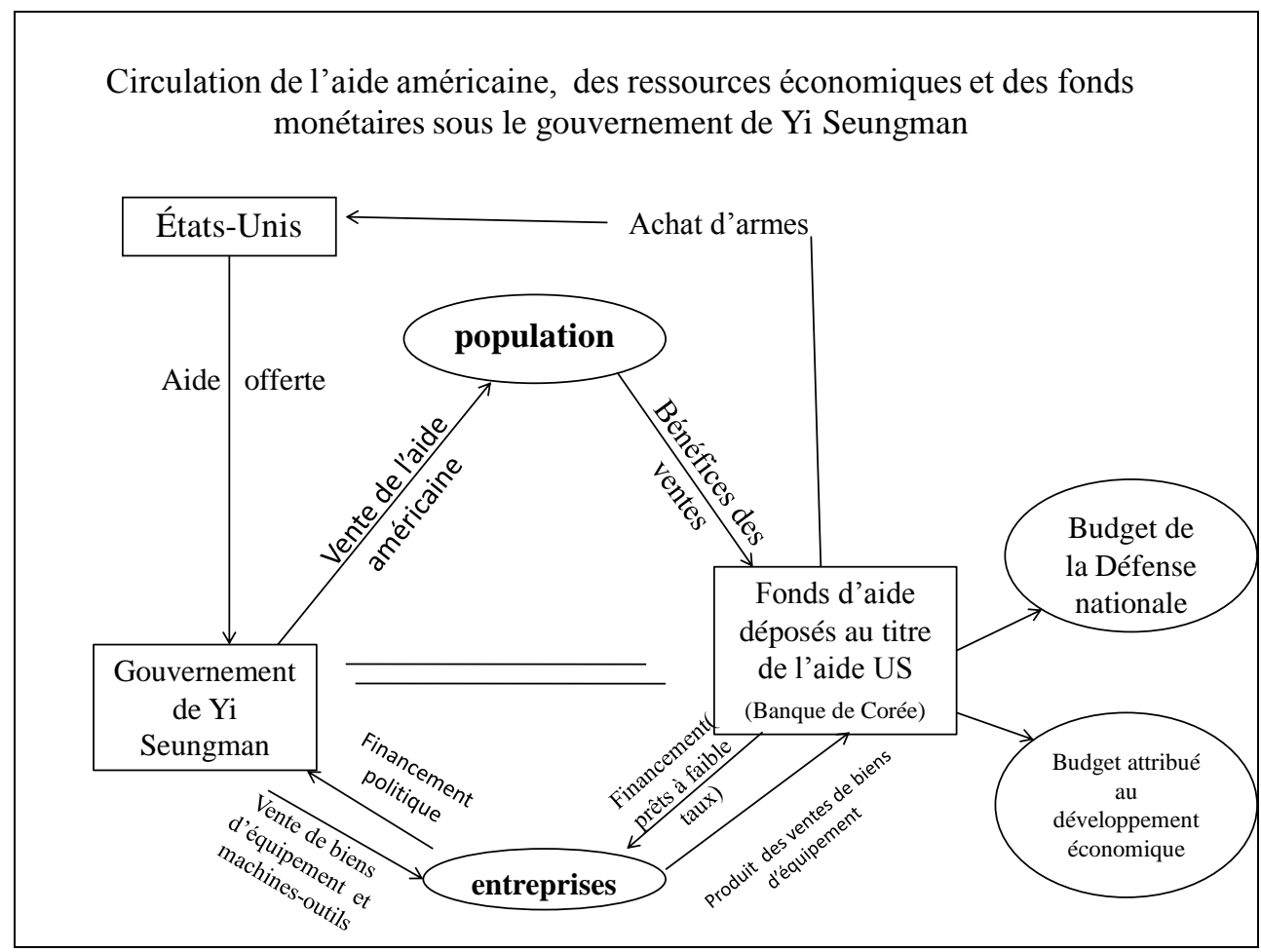

Source: Etabli par nos soins d'après SŎ, Jung-sŏk, Sajin kwa keurim euro poneun han'guk hyŏndaesa, Séoul, Ungjin jisik Hausu, 2005, p. 145.

Calcul, amateurisme, visions à courte vue et atermoiements semblent caractériser un régime présidentiel fort qui s'ccroche au pouvoir, dans la croyance qu'il serait seul capable de diriger le pays.

$\mathrm{Au}$ contraire avec Park Chung-hee, d'ailleurs démarché par les rares grands industriels, la vision des nécessités économiques est plus claire. Là où Yi Sŭngman avait privilégié le textile le sucre et la farine57, Park Chung-hee se lance, à travers plusieurs plans économiques, dans une offensive multi-latérale.

Les plans économiques quinquennaux mettent en avant les priorités et les objectifs. Ainsi en 1962-1966, il s'agit de développer la production électrique, les rendements agricoles et de promouvoir la technologie. De 1967 à 1971, le plan jette les bases de l'industrie lourde, basée sur la pétrochimie et l'acier. Un effort est fait sur les

57 D'où le surnom d'industrie des "trois blancs". 
infrastructures, avec la construction d'autoroutes modernes. C'est aussi dans ce cadre qu'est fondé le POSCO (Pohang Iron \& Steel Co), en 1968, comme préalable au démarrage des chantiers navals et de l'industrie automobile.

Le gouvernement va, dès 1962, s’ingénier à fonder une industrie automobile nationale qui dépassera les productions de l'Argentine ou du Mexique dès $1980^{5}{ }^{8}$. Si la croissance reste importante, les deux chocs pétroliers freinent considérablement le développement. L’inflation grève la consommation intérieure et augmente les coûts de transport.

La normalisation des relations avec le Japon en 1965, le versement de dommages de guerre et d'investissements, permettent d'accélérer les investissements. Dans les années 1970, dans une perspective de désengagement militaire américain en Asie (doctrine Nixon), le président Park investit dans le nucléaire59. Plusieurs centrales sont commandées aux États-Unis et en France, ainsi qu'une usine maréemotrice française, afin de résorber la dépendance énergétique après le choc pétriolier de 1973. En outre, Park Chung-hee a décidé d'orienter l'économie coréenne vers l'exportation et parvient même à inverser la balance des exportations avec les Etats-Unis ${ }^{60}$.

Tab. 7. Balance des importations avec les Etats-Unis, 1960-1980 (\%).

\begin{tabular}{|l|l|l|l|l|l|}
\hline & 1960 & 1965 & 1970 & 1975 & 1980 \\
\hline Exportations vers les USA & 11,1 & 35,2 & 47,3 & 30,2 & 26,3 \\
\hline Importations des USA & 38,9 & 39,3 & 29,5 & 25,9 & 21,9 \\
\hline
\end{tabular}

Source : The Bank of Korea, ECOS DB.

L’une des explications de la réussite de la politique économique du général Park, qui jeta les bases du développement et de la prospérité coréenne tient peut-être à la formation des membres de la junte et des gouvernements successifs, militaires et technocrates, jeunes et bien formés, comprenant les enjeux internationaux autant que nationaux, alors que Yi Sŭngman s'était appuyé sur des partisans qui, pour lui être fidèles, restaient des gens du passé, mal renseignés sur l’industrie et les finances.

${ }^{8}$ GREEN Andrew E. "South Korea's Automobile Industry: Development and Prospects", Asian Survey, Vol. 32, No. 5 (May, 1992), pp. 411-428.

59 HONG Sung-Gul, "The Search for Detterrence: Park's Nuclear Option” in KIM Byung-Kook \& VOGEL Ezra F. (Ed.), The Park Chung-Hee Era, Cambridge (Mass.), Harvard University Press, 2011, pp. 483-510.

$6^{60}$ On ne peut négliger toutefois l'effet de catalyseur de la participation coréenne à la guerre du Vietnam, non seulement du point de vue militaire, mais aussi sur le plan économique. 
Tab. 8. Répartition par âge des membres du Conseil Suprême pour la Reconstruction Nationale (CSRN) et des administrations Yi Sŭngman et Chang Myŏn (\%).

\begin{tabular}{|l|l|l|l|l|l|l|}
\hline & $\begin{array}{l}80 \\
\text { ans }<\end{array}$ & $\begin{array}{l}70-79 \\
\text { ans }\end{array}$ & $\begin{array}{l}60-69 \\
\text { ans }\end{array}$ & $\begin{array}{l}50-59 \\
\text { ans }\end{array}$ & $\begin{array}{l}40-49 \\
\text { ans }\end{array}$ & $\begin{array}{l}30-39 \\
\text { ans }\end{array}$ \\
\hline $\begin{array}{l}\text { Administration Yi Sŭngman } \\
\text { (âges de 1959) }\end{array}$ & 1,4 & 4,7 & 29,1 & 38,5 & 24,3 & 2,0 \\
\hline $\begin{array}{l}\text { Administration Chang Myŏn } \\
\text { (âges de 1960) }\end{array}$ & 0 & 0 & 27,9 & 36,1 & 32,5 & 3,5 \\
\hline $\begin{array}{l}\text { Membres du Conseil Suprême } \\
\text { (âges de 1961) }\end{array}$ & $\mathrm{O}$ & $\mathrm{O}$ & $\mathrm{O}$ & $\mathrm{O}$ & 22,5 & 77,5 \\
\hline
\end{tabular}

Source: PARK, Kisung, Military Authoritarian Regimes and Economic Development: The Rok's Economic Take-Off Under Park Chung Hee, Mémoire du Naval Postgraduate School de Monterey (Ca), 2008.

\section{Conclusions}

La nature du système politique autoritaire qui domina la Corée du Sud reste à analyser objectivement. À l'évidence, nous ne pouvons tout à fait concevoir la Corée du Sud sous les régimes des présidents Yi et Park, comme de nature démocratique. Il faut reconnaître que le pays n’a pas connu, sous ces régimes, de tradition démocratique au sens occidental du terme, puisqu'il est passé directement du joug colonial à la tutelle américaine, puis à un système politique menacé par la sédition et la guerre civile, qui avait cru s'en protéger en édictant en 1948 une mesure normative exceptionnelle, sans songer que cette disposition légale pourrait durer.

Par ailleurs, pour des raisons de bonne politique, intérieure comme extérieure, les deux régimes s'efforcèrent de maintenir une sorte de "formalité démocratique" ou de « démocratie à la coréenne » selon l'expression en vigueur à l'époque de Park, et donc d'organiser des élections. Celles-ci se succèdèrent, sans présenter de grands débats, sauf en 1971, ou bien lors des crises qui amenèrent des modifications normatives. Les élections du temps de Yi Sŭngman restèrent entachées par les fraudes et les intimidations. Le président Yi fut réélu en 1952, 1956 et 1960 grâce à des majorités bien suspectes. Si l'Assemblée nationale continua de fonctionner, elle ne représentait plus un contrepoids au pouvoir exécutif, et se bornait à enregistrer les lois.

Selon Rossiter, les trois crises possibles dans la vie d'une nation démocratique sont : la dépression économique (crise), la rébellion, et la guerre. Or, l’une des crises marquantes des pays en voie de développement semble être, justement le renversement du gouvernement dit «coup d'Etat », et la dérive institutionnelle progressive qui, suspendant ou modifiant en profondeur les institutions et les dispositions normatives, 
conduirait à l'autoritarisme ${ }^{61}$. On peut dire que l'état d'exception, en Corée, représenté par la Loi de sécurité nationale ${ }^{62}$, n’a pas été la suspension du droit, mais la possibilité, en dernier recours, d'invoquer une loi suprême, se surimposant aux autres lois, et même à la Constitution, de facto, sinon de jure. Elle fut largement invoquée pour soutenir des buts politiques, notamment en ce qui concerne les manœuvres de Yi Sŭngman en 1952, avec l'arrestation de députés constitutionnellement protégés par leur statut.

Giorgio Agamben a longuement commenté le principe qui veut que, dans l'état d'exception, la nécessité n'a pas de loi (necessitas legem non habet) ${ }^{63}$. Le péril national ressenti en 1948, faisait que la législation devait être soutenue par une mesure normative exceptionnelle. Ce sera encore le cas après le putsch du général Park en 1961. Dans son recueil d'aphorismes et de maximes, Publius Syrus rappelait ce principe, parfois cruel : Necessitas dat legem, non ipsa accipit (La nécessité donne la loi et ne la reçoit pas).

La nécessité, ressentie par Park Chung-hee, fut à la fois celle de développer l'économie, et celle de devoir, pour cela, empêcher toute contestation. Le « miracle » économique coréen fut une âpre lutte, dans laquelle les droits civiques furent bafoués, toute opposition étant muselée. Nil aliud scit necessitas quam vincere. (La nécessité ne connaît pas autre chose que de vaincre) ${ }^{64}$, disaient les Anciens.

La réussite exemplaire du volontarisme économique de Park Chung-hee, son succès dans la modernisation des campagnes ${ }^{65}$, ont marqué durablement les Coréens. Dans une récente enquête d'opinion, 44\% des sondés ont désigné Park Chung-hee comme le meilleur dirigeant de la Corée depuis 1948, devant (Noh Moo-hyun ${ }^{66}, 24 \%$ ) et Kim Daejung $(14 \%)^{67}$, preuve du charisme intact du général-président ${ }^{68}$ et, peut-être, d'une certaine fascination pour son destin tragique ${ }^{69}$... Etrange réussite en vérité que celle de ce dictateur $^{70}$, proprement illégitime, qui s'attacha à arracher les Coréens à la misère, à établir son pays en tant que puissance, et en retire aujourd'hui une aura d'autant plus grande que nombre de ses successeurs semblent avoir échoué dans bien des domaines.

61 ROSSITER, Clinton L., Constitutional Dictatorship, Crisis Government in Modern Democracies, Princeton (NJ), Princeton Univ. Press, 1948.

${ }^{62}$ En vigueur même sans promulgation de l'état d'urgence.

63 AMGABEN, Giorgio, L'état d'exception, Paris, édition du Seuil, 2003.

64 PUBLIUS SYRUS, Sententiae.

${ }_{65}$ A travers le «mouvement des nouveaux villages » saemaŭl undong.

66 Président de 2003 à 2008.

67 Président de 1998 à 2003.

${ }^{68}$ Enquête réalisée dans toute la Corée du Sud auprès de 2003 personnes du 28 au 30 juillet et du 4 au 6 août 2015 par Han'guk gaellŏp.

${ }^{69}$ Le président Park fut assassiné par son chef de la sécurité en 1979.

70 Il disposait des pleins pouvoirs. 
Avec 3\% des réponses à cette enquête, Yi Sŭngman se retrouve relégué aux côtés du général Chun Doo-hwan ${ }^{71}$, général-président brutal qui commanda la sanglante répression contre le mouvement populaire de Kwangju en $1980^{72}$. Pourtant, Yi Sŭngman possède encore des partisans, en témoigne Ko Yun Sang73 qui conclut son récent article «Yi Sŭngman n'était pas un dictateur »74, en demandant qu'on l'appelle «Père de la Patrie », kungbu.

L'ancien général No Tae-Woo75, promoteur de la transition démocratique et de la Constitution en vigueur aujourd'hui, certes en prison pour corruption aggravée, n'a obtenu que $0,1 \%$ des réponses à l'enquête... Sic transit gloria Coreae.

${ }^{71}$ Chef de la junte, puis président, 1980-1988.

${ }^{72}$ Respectivement à la $4^{\mathrm{e}}$ et à la $5^{\mathrm{e}}$ place du sondage avec $3 \%$ des réponses. Il ne faut pas, négliger l'effet d'éloignement générationnel vis-à-vis de Yi Sŭngman, de plus chassé par une révolution.

73 Professeur de journalisme en université.

74 « Yi Sŭngman-ŭn tokchaecha-ga aniŏtta », jayu kyŏngje-wŏn, chŏlbŭn hamsŏng, no.15-20, 2 avril 2015.

75 Dernier président issu de l'armée, 1988-1993. 


\section{* L'auteur}

Laurent Quisefit est Chercheur associé de l'UMR 8173, Chine, Corée, Japon et chargé de cours à l'INALCO et à l'Université Bordeaux-Montaigne.

URL: < http://www.studistorici.com/progett/autori/\#Quisefit >

\section{Per citare questo articolo:}

QUISEFIT, Laurent, «Autoritarismes civils et militaires en Corée du Sud: 1948-1979», Diacronie. Studi di Storia Contemporanea : Le dittature militari: fisionomia ed eredità politica, 29/12/2015,

URL:< http://www.studistorici.com/2015/12/29/quisefit_numero_24/ >

Diacronie Studi di Storia Contemporanea $\vartheta$ www.diacronie.it

Risorsa digitale indipendente a carattere storiografico. Uscita trimestrale. redazione.diacronie@hotmail.it

Comitato di redazione: Jacopo Bassi - Luca Bufarale - Elisa Grandi - Antonio César Moreno Cantano - Deborah Paci - Fausto Pietrancosta - Alessandro Salvador - Matteo Tomasoni - Luca Zuccolo

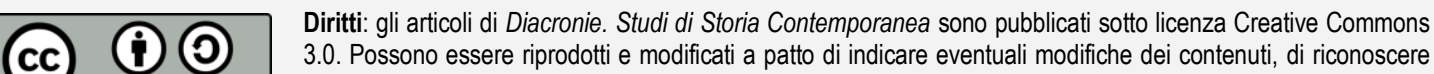

la paternità dell'opera e di condividerla allo stesso modo. La citazione di estratti è comunque sempre autorizzata, nei limiti previsti dalla legge. 